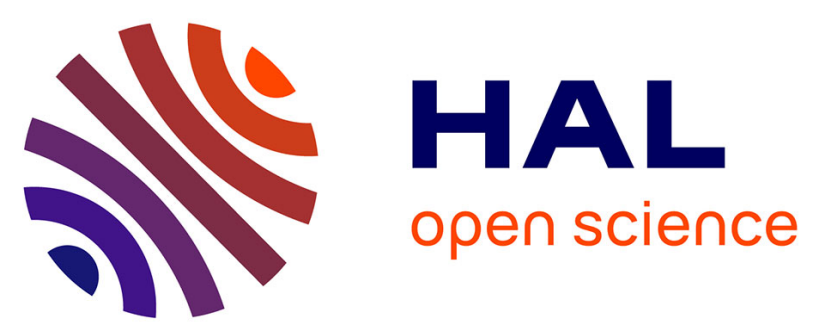

\title{
Changes in Wheelchair Biomechanics Within the First 120 Minutes of Practice: Spatiotemporal Parameters, Handrim Forces, Motor Force, Rolling Resistance and Fore-Aft Stability
}

Nicolas Eydieux, Samuel Hybois, Alice Siegel, Joseph Bascou, Philippe

Vaselin, Hélène Pillet, Pascale Fodé, Christophe Sauret

\section{To cite this version:}

Nicolas Eydieux, Samuel Hybois, Alice Siegel, Joseph Bascou, Philippe Vaselin, et al.. Changes in Wheelchair Biomechanics Within the First 120 Minutes of Practice: Spatiotemporal Parameters, Handrim Forces, Motor Force, Rolling Resistance and Fore-Aft Stability. Disability and Rehabilitation: Assistive Technology, 2020, 15 (3), pp.305-313. 10.1080/17483107.2019.1571117 . hal-02059439

\author{
HAL Id: hal-02059439 \\ https://hal.uca.fr/hal-02059439
}

Submitted on 16 Jun 2020

HAL is a multi-disciplinary open access archive for the deposit and dissemination of scientific research documents, whether they are published or not. The documents may come from teaching and research institutions in France or abroad, or from public or private research centers.
L'archive ouverte pluridisciplinaire HAL, est destinée au dépôt et à la diffusion de documents scientifiques de niveau recherche, publiés ou non, émanant des établissements d'enseignement et de recherche français ou étrangers, des laboratoires publics ou privés. 


\title{
Changes in wheelchair biomechanics within the first 120 minutes of practice: spatiotemporal parameters, handrim forces, motor force, rolling resistance and fore-aft stability
}

\author{
Nicolas Eydieux ${ }^{a, b}$, Samuel Hybois ${ }^{a, b} \mathbb{D}$, Alice Siegel ${ }^{a, b}$, Joseph Bascou ${ }^{a, b} \mathbb{D}_{\text {, }}$ Philippe Vaslinc, Hélène Pillet ${ }^{a} \mathbb{D}^{\mathrm{D}}$, \\ Pascale Fodé $^{b}$ and Christophe Sauret ${ }^{\mathrm{a}}$ (D) \\ ${ }^{a}$ Institut de Biomécanique Humaine Georges Charpak, Arts et Métiers ParisTech, Paris, France; ${ }^{b}$ Centre d'Études et de Recherche sur \\ I'Appareillage des Handicapés Institution Nationale des Invalides, Woippy, France; 'Laboratoire d'Informatique, de Modélisation et d'Optimisation \\ des Systèmes, Université Clermont Auvergne (UCA), Clermont-Ferrand, France
}

\begin{abstract}
Purpose: During manual wheelchair (MWC) skill acquisition, users adapt their propulsion technique through changes in biomechanical parameters. This evolution is assumed to be driven towards a more efficient behavior. However, when no specific training protocol is provided to users, little is known about how they spontaneously adapt during overground MWC locomotion. For that purpose, we investigated this biomechanical spontaneous adaptation within the initial phase of low-intensity uninstructed training.

Materials and methods: Eighteen novice able-bodied subjects were enrolled to perform 120 min of uninstructed practice with a field MWC, distributed over 4 weeks. Subjects were tested during the very first minutes of the program, and after completion of the entire training protocol. Spatiotemporal parameters, handrim forces, motor force, rolling resistance and fore-aft stability were investigated using an instrumented field wheelchair.

Results: Participants rapidly increased linear velocity of the MWC, thanks to a higher propulsive force. This was achieved thanks to higher handrim forces, combined with an improved fraction of effective force for startup but not for propulsion. Despite changes in mechanical actions exerted by the user on the MWC, rolling resistance remained constant but the stability index was noticeably altered.

Conclusion: Even if no indication is given, novice MWC users rapidly change their propulsion technique and increase their linear speed. Such improvements in MWC mobility are allowed by a mastering of the whole range of stability offered by the MWC, which raises the issue of safety on the MWC.

> IMPLICATIONS FOR REHABILITATION

- The learning process of manual wheelchair locomotion induces adaptations for novice users, who change their propulsion technique to improve their mobility.

- Several wheelchair biomechanical parameters change during the learning process, especially wheelchair speed, handrim forces, motor force, rolling resistance and fore-aft stability.

- Fore-aft stability on the wheelchair rapidly reached the tipping limits for users. Technical solutions that preserve stability but do not hinder mobility have to beimplemented, for instance by adding anti-tipping wheels rather than moving the seat forwards with respect to the rear wheels axle.
\end{abstract}

\section{Introduction}

Propelling a manual wheelchair (MWC) requires users to adapt their locomotion technique to their environment, which is not always accessible. In their daily life, users have to repeatedly perform various locomotion tasks such as startup, propulsion, turns or half-spin, on both inclined and level grounds. Such motor tasks engage their upper limbs with different intensities. Some situations and MWC settings result in high joint loading that can potentially lead to upper limb overuse, which may cause pain, injuries and thus reduce the users' quality of life [1]. To identify these drawbacks and try to overcome them, building models and perform experiments during actual MWC locomotion is necessary. Reproducing actual MWC locomotion in laboratory can be achieved by controlling the environment, either with a treadmill or with a stationary ergometer $[2,3]$. However, such protocols may introduce a bias when analyzing individual adaptations. For instance, on a treadmill, the linear velocity of the wheelchair is imposed, and on stationary ergometers, the interaction with the floor (i.e., rolling resistance, slope, etc.) is not reliably reproduced in the absence of haptic feedback. In previous studies, several biomechanical parameters, including cadence, push angle, handrim peak force [4] or propulsion pattern [5], have already been identified as indicators of the occurrence risk of upper limb overuse. In order to prevent these risks, it is essential to identify how they appear during the learning of MWC locomotion. Indeed, the quantification of biomechanical parameters during the learning process could help to understand users' spontaneous adaptation to the use of a MWC. If inexperienced MWC users do not manage to converge towards an optimized propulsion technique, 

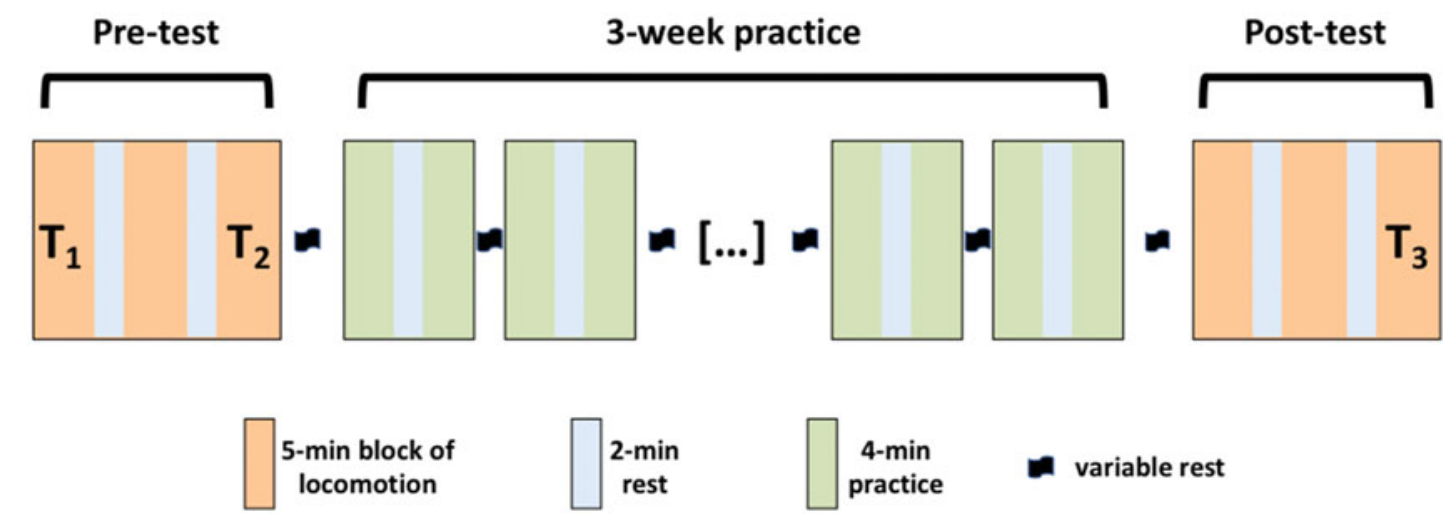

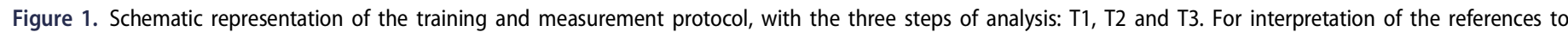
colour in this figure legend, the reader is referred to the web version of this article.

individual assisted training should be reinforced in rehabilitation protocols.

Several studies have already showed that inexperienced subjects quickly and spontaneously adapt their propulsion technique by increasing cycle duration and push angle [6-8]. Significant changes in kinematics and mechanical efficiency have also been found in the case of propulsion on level ground after 7 weeks [9], 80 min [10], and even after only $12 \mathrm{~min}$ of practice [11] without instruction. Nevertheless, it is not ascertained that a self-learning period would yield to similar conclusions for other locomotion tasks or when the study is performed overground rather than on a treadmill [12]. Highly constraining tasks, such as startup or kerb mounting, can require up to three times more energy expenditure than propulsion [13]. However, in spite of its recurrence in daily life, few studies in the literature focused on propulsion and startup [14,15].

Performing overground experiments with a field instrumented MWC appeared necessary to study the evolution of locomotion technique. This ensured that novice users will be placed in genuine locomotion situations of daily life. Moreover, evolutions of both kinematic and kinetic parameters could be taken into account to analyze the effects of MWC practice. The purpose of the current protocol with a field instrumented MWC was also to complete previous results from the literature about MWC learning, by including new parameters describing the interface between users and their environment. Rolling resistance, one of these parameters, was proven to be the main resistance encountered by MWC users at low speeds [16], contributing to users' fatigue. An index of dynamic fore-aft stability has already been proposed [17] to evaluate whether users approach MWC stability limits during propulsion. To the knowledge of the authors, no study considered the evolution of rolling resistance or dynamic stability parameters during the learning process of MWC locomotion. We hypothesized that approaching MWC stability limits would be predictive of the expertise in MWC maneuverability.

The aim of this study was to investigate the evolution in MWC biomechanics during startup and propulsion throughout the learning process of naive able-bodied users, including spatiotemporal parameters, handrim forces, motor force, actual rolling resistance and MWC fore-aft stability. This evolution has been evaluated during the first 120 min of uninstructed practice.

\section{Materials and methods}

\section{Subjects}

Following approval by the relevant ethics committee (CPP Paris VI Pitié Salpêtrière, France no. 2014-A01203-44), 18 able-bodied volunteers (4 females; 14 males) participated in this study. All the subjects were able-bodied subjects, in order to form a homogeneous sample $[10,11,18]$ and because their adaptations would not be influenced by specific muscle weaknesses or lack of control. Subjects' characteristics were: age: 24.9 years old (SD: 2.0; range: 23-37 years old); height: $1.78 \mathrm{~m}$ (SD: $0.05 \mathrm{~m}$, range: $1.63-1.97 \mathrm{~m}$ ); weight: $71.8 \mathrm{~kg}$ (SD: $6.2 \mathrm{~kg}$; range: $60-98 \mathrm{~kg}$ ) and body mass index: $22.6 \mathrm{~kg} / \mathrm{m}^{2}$ (SD: $1.3 \mathrm{~kg} / \mathrm{m}^{2}$, range: $20.0-25.4 \mathrm{~kg} / \mathrm{m}^{2}$ ).

All participants had no prior experience with MWC locomotion and none of them presented any upper limb pain or injury before or during the completion of the study. They were previously informed of the protocol and signed a written informed consent form prior to their participation, allowing both collection and use of their anonymous data for research purposes.

\section{Protocol}

The design of the training program was adapted from the study of Vegter et al. [11] (Figure 1) and consisted in about $120 \mathrm{~min}$ of uninstructed practice distributed over 4 weeks. This program was a low dose and low intensity training program that should prevent from muscular and physiological adaptations. Hence, changes in biomechanical parameters would only reflect neurological adaptations. During the training program, participants were asked to perform 16 min of practice (split into 8 min blocks with at least 1.5 -h in-between) twice a week. During these training sessions, subjects had to move around freely on a hardsmooth level ground, performing startups, forward and backward propulsion, turns and slaloms. Training was performed using four MWC (Otto Bock Voyager; Invacare Kuschall KSL; Vermeiren D200 and Vermeiren Eclips 30) with different characteristics (i.e., geometry, wheel type, mass, and inertia). Subjects were asked to change MWC every $4 \mathrm{~min}$ to prevent getting used to specific MWC configurations and to favor experimentation of different upper limb kinematics and kinetics. Subjects did not receive any information on how to improve their locomotion during the entire training program.

Kinematic and kinetic measurements were carried out before and after the training program, during the initial and final sessions of the protocol. These measurement sessions consisted in three blocks of about $5 \mathrm{~min}$ of field locomotion on a level ground (covered with a low-pile carpet), with 2 min rest between blocks. During the $5 \mathrm{~min}$ of locomotion, participants had to perform a succession of tasks (including startup, propulsion, slalom, and Uturns with various turning radii) at a self-selected comfortable pace. No information was given to the users on the way to perform these tasks. All the subjects used the same wireless 
instrumented MWC (FRET-2, TSR-mesures, France $[16,19])$ during the measurement sessions and both the MWC settings and the tyre pressure were inspected prior to each measurement session.

\section{Measurement procedure}

The FRET-2 allowed measuring the forces and torques applied by the hands on handrims, as well as the forces and torques applied on the seat, the backrest and the footrest. Two angular potentiometers (Spectrol 601-1045, Vishay, USA) allowed the measurement of handrim orientations in the MWC's reference frame, and the calculation of the MWC linear and angular velocities, with the assumption of rolling without sliding on the ground. All these parameters have been sampled at $100 \mathrm{~Hz}$ and the signals of handrim dynamometers have been corrected from baseline values according to procedures detailed elsewhere [20,21].

Reflective markers were also placed on the instrumented MWC (one on each rear wheel center and three other on the chassis) and on both hands of the subject (i.e., second and fifth metacarpal heads, referred to as $\mathrm{MP}_{2}$ and $\mathrm{MP}_{5}$ ) to evaluate the point of application of handrim forces as well as hand patterns [22]. Tridimensional locations of these markers were obtained at $100 \mathrm{~Hz}$ using a 13-cameras optoelectronic motion capture system (Vicon system, hardware: T10 and T20S cameras; Nexus software; Oxford Metrics, Oxford, UK).

Synchronization between the FRET-2 and the motion capture system was ensured by knocking the instrumented handrim of the right wheel with a rubber hammer equipped with a reflective marker at the beginning of each block of locomotion. The shock created a synchronization event noticeable on both the handrim force signal and the hammer marker velocity.

\section{Data processing and computed parameters}

Start-up and propulsion cycles were identified in every block of recorded locomotion with a custom Matlab routine (Matlab, MathWorks, USA). Then, all the parameters described below were computed for each propulsion cycle and startup tasks.

\section{Spatiotemporal parameters}

Once startup and propulsion cycles were isolated, push and freewheeling phases were identified using a two-fold process: (1) automatically, using a 1-Nm threshold on handrims propulsion torques and (2) refined manually considering the resulting forces applied on both handrims. This process allowed the determination of the task time and the durations of both push and freewheeling phases (in seconds and in percentage of the task/ cycle time).

The instantaneous linear velocity of the FRET-2 was calculated based on the rotation of the rear wheels measured by the angular potentiometers and with the assumption that both rear wheels rolled without sliding on the ground (Equation 1). This method allowed the computation of the mean velocity and the intra-task variation of the instantaneous linear velocity.

$$
V_{\mathrm{MWC}}=-\frac{\Delta \theta_{l}+\Delta \theta_{r}}{2 r_{r} \Delta t}
$$

where $V_{\text {MWC }}$ is the "instantaneous" linear velocity of the midpoint between rear wheel centers; $\Delta \theta_{l}$ and $\Delta \theta_{r}$ are the changes in the left and right rear wheel orientations, respectively; $r_{r}$ is the rear wheel radius $(0.31 \mathrm{~m})$ and $\Delta t$ is the time interval (i.e., $0.01 \mathrm{~s}$ in this study). The minus sign before the ratio is necessary because a positive linear velocity is associated to a clockwise rotation of the wheels.

The distance traveled during each startup and propulsion cycle was inferred from the averaged MWC linear velocity and from the task duration.

\section{Hand pattern}

Three-dimensional (3D) data from the motion capture system were used to define, at each instant of the task, the position and the orientation of the MWC reference frame $\left(T_{R_{0} R_{\mathrm{MWC}}}\right)$ with the anteroposterior $X$-axis pointing forward, the inferior-superior $Y$ axis pointing upward and the mediolateral $Z$-axis pointing toward the right [23]. The origin of the MWC reference frame was chosen as the midpoint between rear wheel centers.

The definition of the matrix $T_{R_{0} R_{\mathrm{MWC}}}$ [24] allowed the coordinates of the markers placed on $\mathrm{MP}_{2}$ and $\mathrm{MP}_{5}$ heads to be expressed in the MWC reference frame at each instant of time (Equation 2):

$$
(P)_{R_{\mathrm{MWC}}}=\left(T_{R_{0} R_{\mathrm{MWC}}}\right)^{-1}(P)_{R_{0}}
$$

where $(P)_{R_{M W C}}$ are the homogenous coordinates of the point of interest in the MWC reference frame and $(P)_{R_{0}}$ the homogenous coordinates of the same point in the coordinate system of the motion capture system.

The midpoint between $\mathrm{MP}_{2}$ and $\mathrm{MP}_{5}$ of the subject's right hand was used to define the contact and release angles. These angles were defined by the orientation of the vector joining the wheel center to this midpoint with respect to the $X$-axis of the MWC reference frame, at the beginning and the end of the push phase, respectively. The propulsion angle was then computed by the difference between the contact and release angles.

The 3D trajectory of the midpoint between $\mathrm{MP}_{2}$ and $\mathrm{MP}_{5}$ was projected in the sagittal plane of the MWC reference frame and used to define hand patterns [22] classified as arc (AR), single loop (SL), double loop (DL), and semi-circular (SC).

\section{Handrim kinetics}

Kinetic data obtained from the handrim dynamometers was used to compute the total forces $\left(F_{\text {tot }}\right)$ applied by the hands on both handrims and the propelling torques $\left(T_{\text {prop }}\right)$ along rear wheel rotation axles. For both handrims, the peak and mean values were considered. The mechanical work during both startup and propulsion tasks was calculated from the rotation and the propelling torque of each wheel (Equation 3).

$$
W_{T_{\text {prop }}}=\sum_{i=1}^{n}\left(\Delta \theta_{i} T_{\text {propi }}\right)
$$

where $\Delta \theta_{i}$ is the change in wheel rotation during time interval $i$, $T_{\text {prop } i}$ is the propelling torque during this time interval and $n$ is the number of time intervals during the analyzed task. The point of application of the push force on the handrim was assessed at the midpoint between the markers placed on $\mathrm{MP}_{2}$ and $\mathrm{MP}_{5}$. The knowledge of this point allowed computing the tangential, radial and transversal components of the force applied by the hand on the handrim. Finally, the fraction of effective force (FEF) was assessed as the ratio between the tangential component $\left(F_{\tan }\right)$ and the total push force [25-27]:

$$
\mathrm{FEF}=\frac{F_{\text {tan }}}{F_{\text {tot }}}
$$


Global propulsive force

From both propelling torques, it was possible to determine the global propulsive force $\left(F_{\text {prop }}\right)$ that generated the motion of the MWC:

$$
F_{\text {prop }}=-\frac{T_{\text {propl }}+T_{\text {propr }}}{r_{r}}
$$

where $T_{\text {prop I }}$ and $T_{\text {prop } r}$ are the left and right propelling torques, respectively; and $r_{r}$ is the rear wheel radius. Equation (5) is defined in the case of non-cambered wheels.

\section{Rolling resistance and fore-aft stability}

The normal components of the ground reaction forces applied on front $\left(R_{N f}\right)$ and rear $\left(R_{N r}\right)$ wheels (Equations 6 and 7, respectively) were assessed from the data of all the dynamometers using the mechanical model proposed in [16]. For that purpose, the weight of the FRET-2 $\left(W_{\mathrm{MWC}}=-382.5 \mathrm{~N}\right)$ as well as the anteroposterior position of its center of mass $\left(x_{G}=0.109 \mathrm{~m}\right)$ in the MWC coordinate system were previously determined.

$$
\begin{gathered}
R_{N f}=\frac{\left(x_{A_{s}} F_{S_{y}}-y_{A_{s}} F_{S_{x}}+T_{S_{z}}\right)+x_{G} W_{M W C}}{W_{b}} \\
R_{N r}=-\left(W_{M W C}+F_{S_{y}}+F_{\mathrm{HR}_{y}}+R_{N f}\right)
\end{gathered}
$$

where $x_{A_{S}}$ and $y_{A_{S}}$ are the anteroposterior and inferior-superior coordinates of the chosen point of reduction of the resulting torque applied by the subject on the frame (seat + backrest + footrest); $w_{b}$ is the wheelbase $(0.434 \mathrm{~m}) ; F_{S_{x}}$ and $F_{S_{y}}$ are the anteroposterior and inferior-superior components of the total force applied by the subject on the frame (seat + backrest + footrest); $T_{S_{z}}$ is the transversal torque applied at the chosen point of reduction of the user's mechanical action on the frame $\left(A_{S}\right)$; and $F_{\mathrm{HR}_{\mathrm{y}}}$ is the inferior-superior component of the force applied on both handrims. All the details about the mechanical model are provided in [16].

The normal components of the ground reaction forces $\left(R_{N f}\right.$ and $R_{N r}$ ) were then used to assess the instantaneous rolling resistance and the fore-aft stability index. The rolling resistance (Equation 8) was computed from the model of [16]. The rolling resistance parameter values (i.e., $\lambda_{f}=3.41 \mathrm{~mm}$ and $\lambda_{r}=5.12 \mathrm{~mm}$ for front and rear wheels, respectively) were the ones used in [16], as floor and types of wheel tyres were the same in both experiments. Front $\left(r_{f}\right)$ and rear $\left(r_{r}\right)$ wheels radii were $0.10 \mathrm{~m}$ and $0.31 \mathrm{~m}$, respectively.

$$
F_{\text {roll }}=-\left(\frac{\lambda_{f}}{r_{f}} R_{N f}+\frac{\lambda_{r}}{r_{r}} R_{N r}\right)
$$

Finally, the fore-aft stability was assessed using an index (IS) close to that proposed by [17]:

$$
\mathrm{IS}=\frac{2 R_{N f}}{R_{N f}+R_{N r}}-1
$$

This modification of the previously proposed stability index allowed distinguishing between forward and backward load distribution, where positive values represent higher loads on front wheels and negative values represent higher loads on rear wheels.

\section{Data analysis}

Results have been gathered on the whole population and are presented for the first (T1, 0-5 min of practice) and third (T2, 10-15 min of practice) blocks of the first measurement session, and then for the last block of the second measurement session (T3, 115-120 min of practice, see Figure 1). Results for startup and propulsion are presented separately.

\section{Results}

\section{Spatiotemporal parameters}

Results on spatiotemporal parameters are summarized in Table 1. For both propulsion and startup, the task time increased during the first minutes (from $1.22 \mathrm{~s}$ and $1.91 \mathrm{~s}$ at T1 to $1.37 \mathrm{~s}$ and $1.99 \mathrm{~s}$ at $\mathrm{T} 2$, respectively) and finally returned to their initial values at $\mathrm{T} 3$ (1.22 s and $1.81 \mathrm{~s}$, respectively). During propulsion cycles, the free-wheeling phase followed the same trend as the cycle time. Conversely, for startup, the push time continuously decreased from $\mathrm{T} 1$ to $\mathrm{T} 3$ whereas the free-wheeling phase duration remained constant. When normalized to the cycle time (100\%), the push and free-wheeling phases had similar relative durations in T1 and T3 for propulsion (54/46\%; 52/48\%, respectively) and startup (76/24\%; 72/28\%, respectively).

The mean MWC velocity continuously increased from $0.70 \mathrm{~m} /$ $\mathrm{s}$ at T1 to $1.02 \mathrm{~m} / \mathrm{s}$ at T3 for propulsion, and from $0.33 \mathrm{~m} / \mathrm{s}$ at T1 to $0.51 \mathrm{~m} / \mathrm{s}$ at T3 for startup. The intra-cycle range of the instantaneous linear velocity also increased at T3 $(0.17 \mathrm{~m} / \mathrm{s}$ and $0.60 \mathrm{~m} / \mathrm{s}$, respectively) compared to T1 $(0.24 \mathrm{~m} / \mathrm{s}$ and $0.8 \mathrm{~m} / \mathrm{s}$, respectively) for both propulsion and startup. Throughout the

\begin{tabular}{|c|c|c|c|c|c|c|}
\hline & \multicolumn{3}{|c|}{ Propulsion } & \multicolumn{3}{|c|}{ Start-up } \\
\hline & $\mathrm{T} 1$ & $\mathrm{~T} 2$ & T3 & T1 & $\mathrm{T} 2$ & T3 \\
\hline Cycle time (s) & $1.22(0.22)$ & $1.37(0.28)$ & $1.22(0.23)$ & $1.91(0.3)$ & $1.99(0.33)$ & $1.81(0.28)$ \\
\hline Push time (s) & $0.65(0.10)$ & $0.65(0.11)$ & $0.62(0.10)$ & $1.45(0.25)$ & $1.36(0.18)$ & $1.30(0.15)$ \\
\hline Free-wheeling time (s) & $0.57(0.13)$ & $0.72(0.20)$ & $0.60(0.16)$ & $0.46(0.12)$ & $0.62(0.19)$ & $0.51(0.17)$ \\
\hline Push time $(\% \mathrm{CT})$ & $54(5)$ & $49(6)$ & $52(5)$ & $76(4)$ & $69(5)$ & $72(6)$ \\
\hline Free-wheeling time (\% CT) & $46(5)$ & $51(6)$ & $48(5)$ & $24(4)$ & $31(5)$ & $28(6)$ \\
\hline Cycle distance $(\mathrm{m})$ & $0.85(0.19)$ & $1.18(0.29)$ & $1.26(0.30)$ & $0.64(0.16)$ & $0.91(0.24)$ & $0.94(0.29)$ \\
\hline Mean velocity (m/s) & $0.70(0.11)$ & $0.86(0.13)$ & $1.02(0.12)$ & $0.33(0.06)$ & $0.45(0.07)$ & $0.51(0.10)$ \\
\hline Intra-cycle velocity $(\mathrm{m} / \mathrm{s})$ & $0.17(0.04)$ & $0.21(0.07)$ & $0.24(0.09)$ & $0.60(0.10)$ & $0.77(0.12)$ & $0.88(0.14)$ \\
\hline Contact angle - Left $\left(^{\circ}\right)$ & $94(9)$ & 109 (17) & $113(12)$ & $93(7)$ & $106(17)$ & $108(16)$ \\
\hline Contact angle - Right $\left({ }^{\circ}\right)$ & $93(9)$ & $107(16)$ & $112(13)$ & $90(7)$ & $104(18)$ & 107 (17) \\
\hline Release angle - Left $\left({ }^{\circ}\right)$ & $30(6)$ & $32(7)$ & $29(5)$ & $34(7)$ & $33(6)$ & $30(6)$ \\
\hline Release angle - Right $\left(^{\circ}\right)$ & $31(6)$ & $31(6)$ & $28(5)$ & $35(6)$ & $32(7)$ & $28(5)$ \\
\hline Propulsion angle - Left $\left(^{\circ}\right)$ & $64(10)$ & $77(20)$ & $84(15)$ & $59(10)$ & 73 (19) & 79 (18) \\
\hline Propulsion angle - Right $\left({ }^{\circ}\right)$ & $62(10)$ & $77(20)$ & $84(16)$ & $59(9)$ & $72(20)$ & 78 (19) \\
\hline
\end{tabular}
training program, the distance covered by the MWC continuously increased (from 0.30 to $0.40 \mathrm{~m}$ per cycle) for both tasks (Table 1).

Table 1. Evolution of spatiotemporal parameters during the learning process. 


\section{Hand pattern}

Within the training program, the contact angle noticeably increased between T1 and T2 for both propulsion $\left(94^{\circ}-109^{\circ}\right)$ and startup $\left(93^{\circ}-106^{\circ}\right)$ and continued to slightly increase between T2 $\left(109^{\circ}-113^{\circ}\right.$ and $106^{\circ}-108^{\circ}$, respectively) and T3 (Table 1). The release angle was quite similar between both tasks $\left(30^{\circ}-29^{\circ}\right.$ and $34^{\circ}-30^{\circ}$ ) and was not affected by the training program. Consequently, the propulsion angle drastically increased from $63^{\circ}$ at $\mathrm{T} 1$ to $84^{\circ}$ at $\mathrm{T} 3$ for propulsion and from $59^{\circ}$ at $\mathrm{T} 1$ to $79^{\circ}$ at $\mathrm{T} 3$ for startup. At each step of the learning process, the propulsion angle was slightly lower (about $5^{\circ}$ ) for startup than for propulsion.

Concerning the hand pattern (Table 2), most of the subjects started propelling with an arcing pattern (AR), except two subjects who directly started with a SL pattern. At the end of the first block of locomotion (5 min), four subjects had already evolved toward a SC pattern, whereas half of the subjects kept an arcing pattern. At the end of the first measurement session (15 min), six subjects kept an arcing pattern to propel the MWC whereas three others had already adopted a stable semi-circular hand pattern. At the end of the training program (T3), one

Table 2. Evolution of the handrim patterns during the learning process.

\begin{tabular}{|c|c|c|c|c|c|c|}
\hline \multirow[b]{2}{*}{ Subject } & \multicolumn{3}{|c|}{ Propulsion } & \multicolumn{3}{|c|}{ Start-up } \\
\hline & $\mathrm{T} 1$ & $\mathrm{~T} 2$ & T3 & $\mathrm{T} 1$ & $\mathrm{~T} 2$ & T3 \\
\hline S01 & $\mathrm{AR} / \mathrm{SC}$ & SC & SC & $A R$ & SC & SC \\
\hline S02 & AR & AR & $A R$ & AR & AR & AR \\
\hline S03 & AR & $\mathrm{AR}$ & $S L$ & AR & $\mathrm{AR}$ & $\mathrm{SL}$ \\
\hline S04 & $\mathrm{AR}$ & $\mathrm{AR} / \mathrm{SL}$ & $\mathrm{SC}$ & AR & $\mathrm{AR} / \mathrm{SL}$ & SL \\
\hline S05 & $\mathrm{AR} / \mathrm{SL}$ & $\mathrm{AR} / \mathrm{SL}$ & AR/SL/DL & $A R$ & $\mathrm{AR} / \mathrm{SL}$ & $\mathrm{AR} / \mathrm{SL}$ \\
\hline S06 & $\mathrm{AR}$ & $\mathrm{AR} / \mathrm{SL}$ & AR/SL/DL & $\mathrm{AR}$ & $\mathrm{AR} / \mathrm{SL}$ & AR \\
\hline S07 & $\mathrm{AR} / \mathrm{SL}$ & $\mathrm{SL}$ & $A R$ & $\mathrm{AR} / \mathrm{SL}$ & $\mathrm{AR} / \mathrm{SL}$ & $A R$ \\
\hline S08 & $\mathrm{AR}$ & $A R$ & AR & $\mathrm{AR}$ & $\mathrm{AR}$ & $\mathrm{AR}$ \\
\hline S09 & $\mathrm{AR} / \mathrm{SL}$ & AR/SL/DL & AR/SL/DL & $\mathrm{AR} / \mathrm{SL}$ & $\mathrm{AR} / \mathrm{SL}$ & $\mathrm{AR} / \mathrm{SL}$ \\
\hline S10 & $\mathrm{AR}$ & $A R$ & $A R$ & $\mathrm{AR}$ & $\mathrm{AR}$ & $\mathrm{AR}$ \\
\hline S11 & $\mathrm{AR}$ & $\mathrm{AR}$ & AR & AR & AR & AR \\
\hline S12 & $\mathrm{SL} / \mathrm{SC}$ & $\mathrm{DL} / \mathrm{SC}$ & $\mathrm{SL}$ & $\mathrm{SL} / \mathrm{SC}$ & $\mathrm{DL} / \mathrm{SC}$ & $\mathrm{AR} / \mathrm{SL}$ \\
\hline S13 & AR/SL/SC & $\mathrm{AR} / \mathrm{SC}$ & SC & $\mathrm{AR}$ & $\mathrm{AR} / \mathrm{SC}$ & SC \\
\hline S14 & $\mathrm{AR} / \mathrm{SL}$ & $\mathrm{AR} / \mathrm{SL}$ & SL & $\mathrm{AR} / \mathrm{SL}$ & $\mathrm{AR} / \mathrm{SL}$ & SL \\
\hline S15 & $\mathrm{AR} / \mathrm{SL}$ & $\mathrm{SL}$ & $\mathrm{SL}$ & $\mathrm{AR}$ & $\mathrm{AR} / \mathrm{SL}$ & $\mathrm{AR} / \mathrm{SL}$ \\
\hline S16 & AR & AR & AR & $\mathrm{AR}$ & AR & AR \\
\hline S17 & $\mathrm{AR}$ & SC & SC & $\mathrm{AR} / \mathrm{SC}$ & SC & SC \\
\hline S18 & $\mathrm{SL} / \mathrm{SC}$ & $\mathrm{SL}$ & SL & $\mathrm{AR}$ & SL & SL \\
\hline
\end{tabular}

AR: arcing; DL: double loop; SC: semi-circular; SL: single loop. subject returned from a single loop to an arcing pattern (S07) and another one from a semi-circular to a single loop pattern (S12). Results were globally the same for startup.

\section{Handrim kinetics}

Regarding the handrim forces and torques, peak and mean values of both total push force $\left(F_{\text {tot }}\right)$ and propelling torque $\left(T_{\text {prop }}\right)$ increased noticeably between T1 and T3 (Table 3 ), for both propulsion and startup. The mechanical work of the propelling torque also increased noticeably between T1 and T3 for both propulsion and startup. However, the tangential component $\left(F_{\tan }\right)$ of the handrim forces showed only a moderate increase between T1 and T3. Due to the large increase of the total handrim force and the moderate increase of the tangential component, a decrease of the mean FEF was observed during propulsion throughout the training program. Conversely, FEF increased for startup within the program.

\section{Global propulsive force, rolling resistance, and fore-aft stability}

As a direct result of the increase of the propelling torques, a noticeable increase of the propulsive force was observed between T1 and T3 for both propulsion and startup (Table 3).

Despite the changes in mechanical actions applied by the subjects on the FRET-2, the mean rolling resistance remained globally constant within the training program for both tasks (Table 3), with slightly higher values during startup than during propulsion.

Finally, a higher mean IS was found for startup than for propulsion all along the training program (Table 3 ), indicating a more forward distribution of the ground reaction force. However, when investigating the time course of this index during the tasks, a noticeable increase of the maximal value and a decrease of the minimal value for both startup and propulsion could be observed. Hence, the range of the instantaneous stability index drastically increased between $\mathrm{T} 1$ and $\mathrm{T} 3$ but this evolution differed among subjects. Indeed, some subjects quickly adapted their stability during the first 15 min between T1 and T2, whereas others mostly adjusted between T2 and T3 (Figure 2). At the end of the program, subjects used almost all the range of stability (from -1 to +1 ) during the startup cycle, although the mean value appeared balanced.

Table 3. Evolution of the kinetic and stability parameters during the learning process.

\begin{tabular}{|c|c|c|c|c|c|c|}
\hline & \multicolumn{3}{|c|}{ Propulsion } & \multicolumn{3}{|c|}{ Start-up } \\
\hline & $\mathrm{T}_{1}$ & $\mathrm{~T}_{2}$ & $\mathrm{~T}_{3}$ & $\mathrm{~T}_{1}$ & $\mathrm{~T}_{2}$ & $\mathrm{~T}_{3}$ \\
\hline Peak left propelling torque (Nm) & $-12.0(3.1)$ & $-14.7(4.2)$ & $-16.4(4.2)$ & $-15.9(3.3)$ & $-20.3(3.7)$ & $-22.6(4.3)$ \\
\hline Peak right propelling torque (Nm) & $-11.9(3.0)$ & $-14.8(4.5)$ & $-16.5(4.3)$ & $-15.9(2.6)$ & $-19.8(3.6)$ & $-22.1(4.0)$ \\
\hline Mean left propelling torque ( $\mathrm{Nm})$ & $-5.7(1.2)$ & $-6.6(1.6)$ & $-7.3(1.5)$ & $-9.1(2.3)$ & $-11.5(1.9)$ & $-12.9(2.5)$ \\
\hline Mean right propelling torque (Nm) & $-5.7(1.2)$ & $-6.7(1.8)$ & $-7.5(2.0)$ & $-9.1(1.6)$ & $-11.3(1.86)$ & $-12.8(2.4)$ \\
\hline Mechanical work of the left propelling torque $(J)$ & $8.2(2.5)$ & $12.1(5.3)$ & $14.6(5.2)$ & $12.4(3.4)$ & $19.2(5.9)$ & $23.3(7.7)$ \\
\hline Mechanical work of the right propelling torque $(\mathrm{J})$ & $8.1(2.5)$ & $12.2(6.2)$ & $14.9(5.8)$ & $12.1(3.3)$ & $18.6(5.8)$ & $22.8(6.8)$ \\
\hline Peak left handrim total force $(\mathrm{N})$ & $54.6(14.8)$ & $66.1(16.8)$ & $72.8(17.0)$ & $72.9(15.2)$ & $86.0(16.3)$ & $91.2(16.7)$ \\
\hline Peak right handrim total force $(\mathrm{N})$ & $56.4(16.0)$ & $69.8(20.0)$ & $76.7(20.4)$ & $75.0(14.0)$ & $88.2(19.4)$ & $95.5(20.3)$ \\
\hline Mean left handrim total force $(\mathrm{N})$ & $29.1(6.6)$ & $34.2(7.3)$ & $36.3(6.9)$ & $43.8(10.6)$ & $49.5(9.1)$ & $51.0(10.4)$ \\
\hline Mean right handrim total force $(\mathrm{N})$ & $30.3(7.0)$ & $35.6(8.7)$ & $38.6(8.8)$ & $44.6(9.2)$ & $51.6(10.8)$ & $53.5(10.8)$ \\
\hline Mean left handrim tangential force $(\mathrm{N})$ & $20.5(5.3)$ & $21.6(6.3)$ & $23.1(5.6)$ & $29.5(6.6)$ & $35.2(5.9)$ & $40.0(8.2)$ \\
\hline Mean right handrim tangential force $(\mathrm{N})$ & $20.1(4.6)$ & $21.9(7.4)$ & $23.6(6.1)$ & $31.1(5.3)$ & $36.5(8.3)$ & $41.3(8.9)$ \\
\hline Mean left FEF (\%) & $51(7)$ & $46(9)$ & $44(7)$ & $57(9)$ & $61(7)$ & $67(6)$ \\
\hline Mean right FEF (\%) & $48(6)$ & $44(9)$ & $43(6)$ & $59(8)$ & $61(9)$ & $66(8)$ \\
\hline Peak propulsive force $(\mathrm{N})$ & $76.4(19.8)$ & $94.4(38.0$ & $105.0(26.8)$ & $99.2(18.4)$ & $126.0(22.8)$ & $141.3(27.1)$ \\
\hline Mean propulsive force $(\mathrm{N})$ & $19.7(4.0)$ & $21.1(6.0)$ & $24.8(5.6)$ & $44.0(9.2)$ & $50.8(8.4)$ & $59.4(9.5)$ \\
\hline Mean force of rolling resistance $(\mathrm{N})$ & $9.2(0.4)$ & $9.4(0.5)$ & $9.5(0.5)$ & $10.8(0.6)$ & $10.9(0.7)$ & $10.8(0.9)$ \\
\hline Mean stability index (no unit) & $-0.31(0.15)$ & $-0.27(0.14)$ & $-0.24(0.16)$ & $-0.05(0.12)$ & $0.02(0.13)$ & $0.05(0.16)$ \\
\hline Minimum stability index (no unit) & $-0.80(0.24)$ & $-0.84(0.21)$ & $-0.92(0.24)$ & $-0.76(0.26)$ & $-0.82(0.23)$ & $-0.91(0.23)$ \\
\hline Maximum stability index (no unit) & $0.25(0.11)$ & $0.42(0.18)$ & $0.47(0.20)$ & $0.46(0.12)$ & $0.70(0.17)$ & $0.80(0.26)$ \\
\hline
\end{tabular}



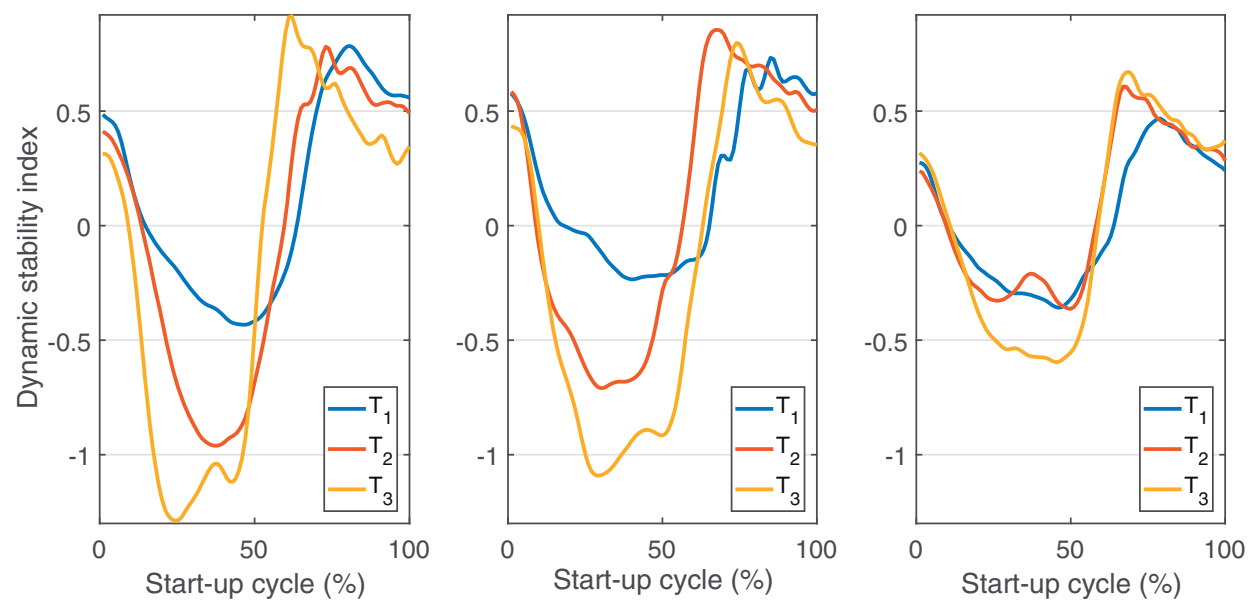

Figure 2. Typical evolutions of fore-aft stability during start-up for 3 different subjects of the protocol. For interpretation of the references to colour in this figure legend, the reader is referred to the web version of this article.

\section{Discussion}

\section{Spatiotemporal parameters}

For propulsion, the push time remained constant between T1 and $\mathrm{T} 2$ and slightly decreased between $\mathrm{T} 2$ and $\mathrm{T} 3$. This trend can be interpreted as coming from the changes in both MWC linear velocity and propulsion angle, which increased concomitantly between $\mathrm{T} 1$ and $\mathrm{T} 2$. This resulted in a constant push time despite the increase of the hand velocity, which was inferred from the increase of the wheel angular velocity. Between T2 and T3, the increase of the propulsion angle was limited whereas the mean MWC linear velocity increased noticeably, which logically resulted in a decrease of the push time. For startup, the increase of the hand velocity was such that despite an increase of the propulsion angle between $\mathrm{T} 1$ and $\mathrm{T} 2$, the push time continuously decreased.

For both propulsion and startup, the free-wheeling time increased between $\mathrm{T} 1$ and $\mathrm{T} 2$ then returned to the initial values at T3. In the meantime, the standard deviation of the free-wheeling time was also higher, potentially due to the variation in hand patterns between the subjects. Subsequently to the evolutions of push and free-wheeling duration, the cycle time increased between $\mathrm{T} 1$ and $\mathrm{T} 2$ and then decreased between $\mathrm{T} 2$ and $\mathrm{T} 3$.

Compared to the results of Vegter et al. [11], cycle time, push time and free-wheeling time were noticeably higher in this study. The difference in push time can be explained by the method used to define the push phase [28]: it was based on the propelling torque for Vegter et al. [11] and on the total force in this study. Basically, in our population, this difference ranged between 0.05 and $0.10 \mathrm{~s}$. The differences in push and cycle time can also be explained by the protocol. Indeed, the study of Vegter et al. [11] was performed on a treadmill. This has the benefit to allow control of the required power output, but with an imposed and controlled velocity of $1.11 \mathrm{~m} / \mathrm{s}$. This velocity was noticeably higher than the self-selected velocity chosen by our subjects for overground locomotion. The recent publication of Chenier et al. [12] on the comparison between overground and treadmill MWC propulsion suggested that the velocity imposed in the study of Vegter et al. [11] could be greater than the one subjects would have intuitively selected. Hence, treadmill and overground experiments should be seen as complementary. Considering the above remarks, it is difficult to precisely compare time parameters between studies performed on a treadmill and overground. However, it can be assumed that actual overground conditions are more ecological than experiments performed on a treadmill.

\section{Hand patterns}

Regarding the hands, the angular sector covered by the hands on the handrim during each cycle (i.e., propulsion angle) increased with practice by the mean of a more backward grip of the rim while maintaining a constant release angle. This evolution was similar to that described in [11], even if the propulsion angles were noticeably higher in this study (from $10^{\circ}$ to $20^{\circ}$ ). This difference can once again be explained by both the difference in push phase identification and the use of a treadmill compared to overground locomotion. Indeed, the difference could partly be explained by the urgency of grasping the handrim earlier during the free-wheeling phase, for safety purposes, when propelling on a treadmill [12]. This spontaneous increase of the push duration is in accordance with the general recommendations for efficient MWC propulsion [29].

Although arcing hand pattern is associated with the behavior of novice wheelchair users, only a minority of subjects kept an arcing strategy throughout the learning protocol. Most users spontaneously evolved toward SC or SL patterns, which have been shown to be energetically more efficient [22,30]. Besides, even if semi-circular patterns were generally associated to expert MWC users [22], this strategy was adopted by some subjects in this study after only a few minutes of practice ( $<15 \mathrm{~min})$.

\section{Handrim kinetics}

As it was observed by Vegter et al. [11], subjects rapidly increased the force they applied on the handrims during propulsion, thus creating a higher propelling torque. In our study, this trend was observed in both startup and propulsion tasks and it explained the concomitant increase of intra-cycle variations of the linear velocity. Compared to Vegter et al. [11], mean total forces during propulsion were lower in this study whereas peak values were globally similar. The higher mean value of total forces in Vegter et al. [11] could be explained by the higher velocity but also by the lower push time in their experiments.

The mean FEF showed a constant increase for startup, but a decrease for propulsion throughout the learning process. This result can be explained by the greater handrim angular velocity due to the increase of the MWC linear velocity - during propulsion than during startup cycles, making it difficult for the subjects to grasp the handrim at the beginning of the push phase. This behavior generally resulted in a negative instantaneous tangential 
force [28], and thus in a negative FEF value. For startup, conversely, subjects were able to optimize their push phase because the wheels, and thus the handrims, were motionless at the beginning of each cycle.

Mean FEF values found in this study were lower than previous values found for propulsion [11,31]. However, different computation methods have been used in these studies. Indeed, Lenton et al. [31] calculated the tangential component of the handrim from the ratio between the propelling torque and the handrim radius. This method tends to over-estimate the tangential force since it neglects the local torque applied by the hand at the contact point [32]. In addition, the mean FEF can be calculated either by the ratio between the mean values of tangential and total forces, or by the mean of the instantaneous FEF ratio. The latter method was chosen in the present study but tends to under-estimate the mean FEF. Indeed, at the beginning of the push phase, the tangential force can be negative and the total force low, resulting in a high negative value of FEF. These methodological differences may explain the variations of FEF between studies and limit the comparison of this parameter.

\section{Global propulsive force, rolling resistance and fore-aft stability}

As a direct consequence of the increase of handrim propelling torques, the propulsive force increased throughout the training program followed by the subjects in this study, for both propulsion and startup tasks. Due to changes in forces applied on handrims, changes in rolling resistance throughout the training program could have been expected. However, for each tested subject, rolling resistance remained stable between $\mathrm{T} 1, \mathrm{~T} 2$, and $\mathrm{T} 3$ for both propulsion and startup tasks. However, even if there was no change in the mean rolling resistance, the instantaneous intratask values of this parameter were altered. Also, a difference of $+10 \%$ was found in the mean rolling resistance from propulsion to startup, resulting from the subject's mechanical actions on the FRET-2 (handrim, seat, footrest, and backrest). This result demonstrates the necessity of being cautious when calculating the mechanical power from mean velocity and rolling resistance, because both parameters evolved during the tasks. This method was however commonly applied in previous studies [33,34]. Fortunately, its effect seems to be limited with respect to the evaluated factors and conclusions should thus not be challenged.

The stability index was also influenced by practice. Indeed, if the mean values were not noticeably altered, the range of instantaneous values demonstrated that with practice subjects did use the whole range of stability (from -1 to +1 ) and even started to manage startup and propulsion with slight tipping hazards. Indeed, the tipping hazard drastically increased, backwards during the push phase and forwards during the free-wheeling phase. The changes in forces exerted on the seat and the backrest during locomotion were responsible for this change in fore-aft stability. Finally, it revealed that subjects spontaneously favored mobility at the expense of stability.

\section{Limitations}

The main limitation of this study was that it has been conducted among a population of able-bodied subjects with no prior MWC use in order to eliminate any bias of MWC experience. Thus, transferring the findings of this study to genuine MWC users should be done cautiously, especially for subjects with pathologies impacting motor control. Indeed, strategies spontaneously adopted by such users may be guided by different objectives than those of able-bodied subjects. Moreover, conclusions about the evolution of upper limbs loading with practice cannot directly be inferred only from the results of handrim kinetics. It requires an inverse dynamic analysis, in order to take into account the motion of the upper extremities, and this kind of analysis revealed different behaviors depending on subjects [35]. Finally, startup and propulsion are common tasks in the daily use of a MWC, but skill acquisition and locomotion evolution with practice should also be studied in other specific tasks, such as U-turns or wheelies [36].

\section{Conclusion}

The subjects who participated in this study rapidly modified their behavior on the MWC during locomotion, through a self-learning process. Indeed, they reached higher MWC linear velocities, thanks to a higher propulsive force. The increase in the force they applied on the handrim was concomitant with an increase in the anteroposterior force on the frame that altered the fore-aft stability of the MWC. This behavior raised the issue of safety on the MWC, since it appeared that users gained confidence very quickly. A conventional solution would be to recommend moving the seat forwards with respect to the rear wheels [37]. However, this adjustment will induce a higher rolling resistance and limit users' mobility, which in turn would require a higher load on users' upper limbs [38]. An alternative solution could be to lower the seat and the backrest in order to limit the effect on MWC fore-aft stability of the anteroposterior force applied by the subject [16]. Furthermore, the environment must be considered before such adjustments are made since it can lead to an increase of the shoulder load during ramp ascent [39], for instance. Another solution could be to keep the same MWC settings but to add antitipping wheels, which would provide an adequate compromise between user's mobility and MWC fore-aft stability.

\section{Disclosure statement}

The authors report no conflict of interest.

\section{ORCID}

Samuel Hybois (D) http://orcid.org/0000-0003-3838-2860

Joseph Bascou (D http://orcid.org/0000-0001-5290-8158

Hélène Pillet (iD http://orcid.org/0000-0002-0263-5910

Christophe Sauret (iD http://orcid.org/0000-0002-6196-2698

\section{References}

[1] Kemp BJ, Bateham AL, Mulroy SJ, et al. Effects of reduction in shoulder pain on quality of life and community activities among people living long-term with $\mathrm{SCl}$ paraplegia: a randomized control trial. J Spinal Cord Med. 2011;34: 278-284.

[2] Gorce P, Louis N. Wheelchair propulsion kinematics in beginners and expert users: influence of wheelchair settings. Clin Biomech. 2012;27:7-15.

[3] van der Woude LHV, Bouw A, van Wegen J, et al. Seat height: effects on submaximal hand rim wheelchair 
performance during spinal cord injury rehabilitation. J Rehabil Med. 2009;41:143-149.

[4] Rankin JW, Kwarciak AM, Richter WM, et al. The influence of wheelchair propulsion technique on upper extremity muscle demand: A simulation study. Clin Biomech. 2012;27: 879-886.

[5] Rodgers MM, Keyser RE, Gardner ER, et al. Influence of trunk flexion on biomechanics of wheelchair propulsion. J Rehabil Res Dev. 2000;37:283-295.

[6] De Groot S, Veeger DHEJ, Hollander AP, et al. Wheelchair propulsion technique and mechanical efficiency after $3 \mathrm{wk}$ of practice. Med Sci Sports Exerc. 2002;34:756-766.

[7] Goosey-Tolfrey VL, West M, Lenton JP, et al. Influence of varied tempo music on wheelchair mechanical efficiency following 3-week practice. Int J Sports Med. 2011;32: 126-131.

[8] Yao WX, Cordova A, De Sola W, et al. The effect of variable practice on wheelchair propulsive efficiency and propulsive timing. Eur J Phys Rehabil Med. 2012;48:209-216.

[9] de Groot S, de Bruin M, Noomen SP, et al. Mechanical efficiency and propulsion technique after 7 weeks of lowintensity wheelchair training. Clin Biomech (Bristol, Avon). 2008;23:434-441.

[10] Vegter RJK, Lamoth CJ, de Groot S, et al. Inter-individual differences in the initial 80 minutes of motor learning of handrim wheelchair propulsion. PLoS One. 2014;9:e89729.

[11] Vegter RJK, de Groot S, Lamoth CJ, et al. Initial skill acquisition of handrim wheelchair propulsion: a new perspective. IEEE Trans Neural Syst Rehabil Eng. 2014;22:104-113.

[12] Chénier F, Champagne A, Desroches G, et al. Unmatched speed perceptions between overground and treadmill manual wheelchair propulsion in long-term manual wheelchair users. Gait Posture. 2018;61:398-402.

[13] Sauret C, Vaslin P, Dumas R, et al. Computation of the mechanical power of a manual wheelchair user in actual conditions: preliminary results. Comput Methods Biomech Biomed Eng. 2012;15:173-174.

[14] Freixes O, Fernández SA, Gatti MA, et al. Wheelchair axle position effect on start-up propulsion performance of persons with tetraplegia. J Rehabil Res Dev. 2010;47:661-668.

[15] Boninger ML, Baldwin M, Cooper RA, et al. Manual wheelchair pushrim biomechanics and axle position. Arch Phys Med Rehabil. 2000;81:608-613.

[16] Sauret C, Vaslin P, Lavaste F, et al. Effects of user's actions on rolling resistance and wheelchair stability during handrim wheelchair propulsion in the field. Med Eng Phys. 2013;35:289-297.

[17] Sauret C, Vaslin P, Bascou J, et al. Proposal of an index for evaluating pitch instability during actual locomotion with a manual wheelchair. Comput Methods Biomech Biomed Eng. 2013;16:130-131.

[18] Vegter RJK, Hartog J, de Groot S, et al. Early motor learning changes in upper-limb dynamics and shoulder complex loading during handrim wheelchair propulsion. J Neuro Eng Rehabil. 2015;12:26.

[19] Dabonneville $M$, Vaslin $P$, Kauffmann $P$, et al. A self-contained wireless wheelchair ergometer designed for biomechanical measures in real life conditions. Technol Disabil 2005;17:63-76.

[20] Sauret C, Dabonneville $M$, Couétard $Y$, et al. Zeroing of sixcomponent handrim dynamometer for biomechanical studies of manual wheelchair locomotion. Comput Methods Biomech Biomed Eng. 2014;17:416-422.

[21] Sauret C, Couetard Y, Vaslin P. Dynamic calibration of a wheelchair six-component wheel dynamometer rolling on the floor. Comput Methods Biomech Biomed Eng. 2011;14: 67-69.

[22] Boninger ML, Souza AL, Cooper RA, et al. Propulsion patterns and pushrim biomechanics in manual wheelchair propulsion. Arch Phys Med Rehabil. 2002;83:718-723.

[23] Cooper RA, DiGiovine $C P$, Boninger $M L$, et al. Filter frequency selection for manual wheelchair biomechanics. J Rehabil Res Dev 2002;39:323-336.

[24] Legnani G, Casolo F, Righettini $P$, et al. A homogeneous matrix approach to 3D kinematics and dynamics - I. Theory. Mech Mach Theor. 1996;31:573-587.

[25] Boninger ML, Cooper RA, Baldwin MA, et al. Wheelchair pushrim kinetics: body weight and median nerve function. Arch Phys Med Rehabil. 1999;80:910-915.

[26] Dallmeijer AJ, van der Woude LHV, Veeger DHEJ, et al. Effectiveness of force application in manual wheelchair propulsion in persons with spinal cord injuries. Am J Phys Med Rehabil. 1998;77:213-221.

[27] Lin C-J, Lin P-C, Su F-C, et al. Biomechanics of wheelchair propulsion. J Mech Med Biol. 2009;9:229-242.

[28] Kwarciak AM, Sisto SA, Yarossi M, et al. Redefining the manual wheelchair stroke cycle: identification and impact of nonpropulsive pushrim contact. Arch Phys Med Rehabil. 2009;90:20-26.

[29] Paralyzed Veterans of America Consortium for Spinal Cord Medicine. Preservation of upper limb function following spinal cord injury - a clinical practice guideline for healthcare professionals. J Spinal Cord Med 2005;28:434-470.

[30] Slowik JS, Requejo PS, Mulroy SJ, et al. The influence of wheelchair propulsion hand pattern on upper extremity muscle power and stress. J Biomech. 2016;49:1554-1561.

[31] Lenton JP, van der Woude LH V, Fowler NE, et al. Hand-rim forces and gross mechanical efficiency at various frequencies of wheelchair propulsion. Int J Sports Med. 2013;34: 158-164.

[32] Sabick MB, Zhao KD, An KN. A comparison of methods to compute the point of force application in handrim wheelchair propulsion: a technical note. J Rehabil Res Dev. 2001; 38:57-68.

[33] de Groot S, Vegter RJK, van der Woude LHV. Effect of wheelchair mass, tire type and tire pressure on physical strain and wheelchair propulsion technique. Med Eng Phys. 2013;35:1476-1482.

[34] Mason BS, Lemstra M, van der Woude LHV, et al. Influence of wheel configuration on wheelchair basketball performance: wheel stiffness, tyre type and tyre orientation. Med Eng Phys. 2015;37:392-399.

[35] Hybois S, Siegel A, Bascou J, et al. Shoulder kinetics during start-up and propulsion with a manual wheelchair within the initial phase of uninstructed training. Disabil Rehabil Assist Technol. 2017;19:1-10.

[36] Kirby RL, Smith C, Seaman R, et al. The manual wheelchair wheelie: a review of our current understanding of an important motor skill. Disabil Rehabil Assist Technol. 2006; 1:119-127. 
[37] Hong E-K, Pearlman J, Salatin B, et al. Design and development of a lightweight, durable, adjustable composite backrest mounting. Assist Technol. 2011;23:24-35.

[38] Mulroy SJ, Newsam CJ, Gutierrez DD, et al. Effect of fore-aft seat position on shoulder demands during wheelchair propulsion: part 1. A kinetic analysis. J Spinal Cord Med. 2005;28:214-221.

[39] Kotajarvi BR, Sabick M, Basford J, et al. The effect of wheelchair seat position on peak shoulder joint moments during propulsion up an incline. Arch Phys Med Rehabil. 2003;84:E30. 